\title{
Long-term cognitive deficits in patients after surgery on aneurysms of the anterior communicating artery
}

\author{
Lynley M Stenhouse, Robert G Knight, Barry E Longmore, Samir N Bishara
}

\begin{abstract}
The long term recovery of a series of 27 patients who had undergone rupture and repair of an aneurysm of the anterior communicating artery was assessed using a number of neuropsychological measures. Testing took place 12 to 84 months post surgery. On the basis of the results from tests of intellect, memory, and conceptual learning, three subgroups of patients were identified: those with persuasive cognitive defects, those with evidence of residual frontal lobe damage and those who showed no evidence of dysfunction. The occurrence of cerebral vasospasm was the most consistent predictor of long term cognitive deficit.
\end{abstract}

Patients surviving an aneurysmal subarachnoid haemorrhage (SAH) with no clinical signs of neurological impairment may, however, show evidence of cognitive dysfunction on standardised neuropsychological tests. ${ }^{1-7}$ For example, Ljunggren et al $t^{6}$ found neuropsychological deficits in $83 \%$ of patients tested an average of 3.5 years after an $\mathrm{SAH}$, with memory loss being the most common deficit. Although estimates of the incidence of persisting deficits in cognition are lower in other studies, ${ }^{27}$ it is apparent that detectable cognitive impairment is a common result of SAH. The nature and severity of neuropsychological deficit is dependent on the artery affected. Ruptures of aneurysms of the internal carotid or middle cerebral artery frequently cause pronounced aphasic, spatial, and memory impairments. ${ }^{1}$ Aneurysmal rupture involving the anterior communicating artery frequently results in persistent memory problems and personality changes. ${ }^{5-16}$ In particular, severe amnesic deficits have been reported in patients who have had surgical repair of ruptured aneurysms arising from the anterior communicating artery (ACoA)..$^{10-16}$

As well as being a common site for the development of aneurysms, the ACoA is notable for the considerable number of anomalous variations to normal anatomy that occur. ${ }^{1718}$ In addition, numerous small perforating arteries arise from the ACoA. The preservation of these presents the neurosurgeon with a considerable challenge, even though the application of microsurgical techniques and other advances in neurosurgical techniques and in neuroanaesthesia, have substantially reduced the incidence of mortality and morbidity. ${ }^{11}$

Neurological damage following ACoA aneurysm rupture may be a consequence of ischaemia and infarction in the territories of the anterior cerebral artery or the ACoA perforating branches, or raised intracranial pressure from intracerebral bleeding, brain swelling, or hydrocephalus. ${ }^{19}$ Ischaemia and infarction are often a consequence of cerebral vasospasm, a common pre- or postoperative complication. ${ }^{20}$ It is not surprising therefore that survivors of the rupture and consequent surgery do not always return to their premorbid level of functioning and may be left with profound changes in their personality or cognition. The global amnesic syndrome, which has been reported in some patients surviving ACoA aneurysm rupture, has been of particular interest. ${ }^{1014-16}$ The principal reason for this is that the amnesia appears to be a sequel of damage to structures in the basal forebrain, ${ }^{13}$ an important segment of the diencephalic-limbic memory circuit proposed by Mishkin et al. ${ }^{2122}$ The pattern of amnesic deficits is similar in quality to that shown by alcoholic Korsakoff patients. In particular, both alcoholic Korsakoff and ACoA aneurysmal amnesics show a greater incidence of confabulation, and dysfunction on memory/ learning tasks associated with frontal lobe damage (such as conceptual learning, recency discrimination, and temporal sequencing), than those with global amnesia with lesions primarily confined to limbic structures in the temporal lobes. ${ }^{101415}$ It is apparent also that patients recovering from ACoA aneurysm rupture may show signs of intellectual deterioration and deficits on tasks measuring putative frontal lobe functions, independently of, or in addition to their memory deficits.

Although psychometric data on ACoA aneurysm rupture patients are available, results tend to be presented for subjects who have been selected because they have established cognitive deficits, ${ }^{101423}$ many of whom are tested in the first few weeks or months after surgery. An exception are two studies of the incidence and severity of persistent intellectual and memory deficits in patients surviving a rupture of an ACoA aneurysm. ${ }^{24}{ }^{25}$ Neither study, however, incorporated an age matched control group, nor were tests likely to be specifically sensitive to frontal damage included in the assessment battery. Our aim was to report the incidence of cognitive deficits in a series of 27 unselected patients tested 
more than 12 months after surgical repair of an ACoA aneurysm rupture and, where possible, to relate such deficits to pre- and postoperative variables.

\section{Patients and methods}

The files of all patients who had undergone rupture and surgical repair of an $A C o A$ aneurysm at the neurosurgical unit of Dunedin Hospital between August 1980 and August 1987, were reviewed. Of the total of 47 patients who had had surgery, two had subsequently died (one postoperatively and the other of carcinoma of the colon 3.5 years after the operation). A further 15 patients were excluded from follow up for reasons which included: premorbid history of psychiatric disturbance or mental retardation, deafness, age more than 70 years at the time of follow up, or were no longer able to be contacted. Letters were sent to the remaining 30 patients, inviting them to take part in the study. A total of 27 patients aged between 33 and 70 years agreed to participate. The average (SD) age of the patients was $55.2(10 \cdot 7)$ years; 13 were male, 14 were female. They had an average of $10.42(1.25)$ years formal school education. The average length of time since surgery at follow up was $54 \cdot 2$ months (range $=12$ to 84 months). All patients were graded at the time of operation using the Hunt and Hess scale. ${ }^{26}$ The preoperative grades were I for seven patients, II for 15, and III for five patients. Essentially, the same operation was performed for all 27 subjects by the same neurosurgeon (SNB). The side of approach was on the right for 14 patients, and on the left for 13 patients. The neck of the aneurysm was satisfactorily clipped in each case.

A control group of 20 volunteers with no history of neurological or psychiatric disorder was recruited from among the spouses of the patients and from local service groups. This group was matched for age [average $55 \cdot 1$ (10.99) years], and years of school education [average 10.5 (1.09) years]. There were 10 males and 10 females in the sample. Both groups were also matched for performance on the National Adult Reading Test (NART). ${ }^{27}$ This is a measure comprising 50 words of irregular pronunciation (for example, thyme, demesne), which the subject is requested to read aloud. The number of pronunciation errors provides an index used to estimate premorbid IQ level. The two groups did not differ in their average NART estimated IQ scores (table 1), $\mathrm{t}=0.66, \mathrm{p}=0.3$, implying that the matching for premorbid cognitive/ educational factors was successful.

During the testing session, all subjects completed the following measures: Four subtests of the Wechsler Adult Intelligence Scale (WAIS; Picture Arrangement, Block Design, Vocabulary, and Arithmetic ${ }^{28}$, the Wechsler Memory Scale ${ }^{29}$ (WMS), the NART, and the Wisconsin Card Sorting Test ${ }^{30}$ (WCST). For each subject, a Full Scale IQ (FSIQ) based on the 4-subtest short-form of the WAIS and a WMS Memory Quotient (MQ), were calculated.

\section{Results}

Means and standard deviations for neuropsychological tests are presented in table 1 . These results are based on data from 25 patients; two of the 27 subjects were unable to complete the tests. One patient (case 26) was unwilling to attempt some tests. Time constraints prevented completion of testing of the second patient (case 9). The individual results of the 27 aneurysm patients are discussed in more detail below. Of the 25 patients included in the group data, four patients (cases 18, 19, 20 and 27) were unable to attempt all the tests used. The WAIS could not be administered to case 18 because of severe visual impairment. Cases 19 and 20 were unable to carry out the WCST because the severity of their cognitive impairment was such that they could not understand the test instructions. Case 27 was so severely impaired that she could attempt only the NART and the WMS.

The WAIS IQ scores did not significantly discriminate between the two groups, $\mathrm{t}=1.53, \mathrm{p}<0.07$, although the patient group's mean score was some five points lower than that of the comparison group. The difference between the premorbid IQ estimate provided by the NART and the current IQ estimate obtained from the WAIS, was calculated for each subject for use as an index of intellectual deterioration. The mean IQ deterioration scores (table 1) of the surgical and control groups were significantly different, $t=2.56, p<0.01$. A deterioration score of 15 (one SD of the IQ scale) is generally accepted as suggesting clinically significant deterioration. In this case, the mean deterioration score of the patient group, which was 4.6 IQ points, lay well above the cut off score of 15 . On average

Table 1 Means and standard deviations of the neuropsychological test scores

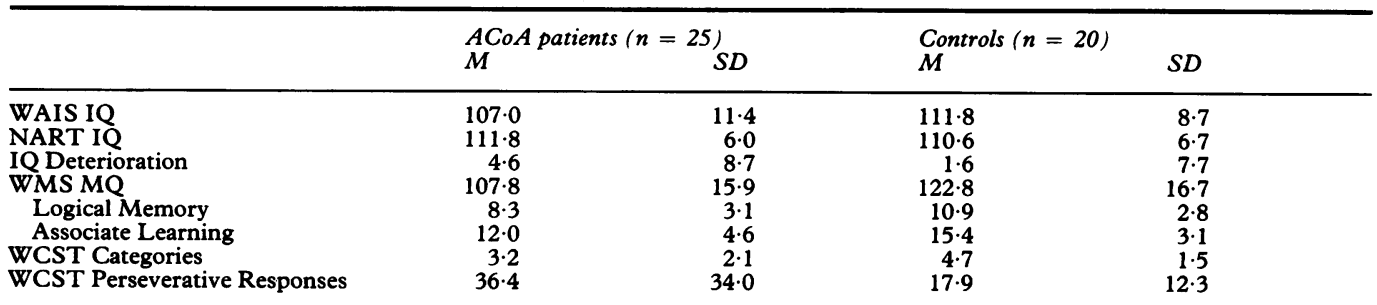

Note: WAIS - Wechsler Adult Intelligence Scale; NART - National Adult Reading Test; WMS - Wechsler Memory Scale; WCST - W isconsin Card Sorting Test. 
therefore the surgical group did not show evidence of a clinically significant level of dementia using this particular index.

On the WMS, the between group MQ score difference was significant, $t=3.06, p<0.01$, as were the differences on the two subtests of the WMS measuring verbal memory: Logical Memory, $\mathrm{t}=2.93, \mathrm{p}<0.01$, and Associate Learning, $t=2.82, p<0.01$. On the WCST, the aneurysm patients made significantly fewer successful category shifts, $\mathrm{t}=2.44, \mathrm{p}<0.01$, and more perseverative responses, $t=2 \cdot 34$, $\mathrm{p}<0.01$.

The group mean scores, however, disguise important individual differences among the aneurysm patients. Results from individual cases were therefore examined in more detail. For each neuropsychological measure, criteria for test performance strongly indicating impairment were formulated as follows: intellectual deterioration was defined as a difference score between the NART IQ and WAIS IQ of 15 or more points (that is, greater than one standard deviation on the IQ scale). A total of five surgical patients, but no control subjects, met this criterion for intellectual deterioration. Memory impairment was defined as either a difference between NART IQ and WMS IQ of 15 or more points or an absolute MQ score lower than 85 (one standard deviation below the MQ mean of 100). Seven aneurysm patients met one or both of these standards. One control subject had a NART IQ-MQ difference of 16 points. Finally, a pathological score on the WCST was defined as either a failure to learn three or more categories or the commission of 30 or more perseverative responses. One control subject scored in the pathological range on both criteria (learning two categories and making 34 perseverative responses), and a further five learned at least three categories, but made more than 30 per- severative errors. Eleven surgical patients performed below the cut off standards, 10 failing on both criteria. A further three patients were unable to attempt the test, as mentioned previously, because of their degree of cognitive impairment.

In table 2 , we present the individual test scores for the 27 surgical patients. Scores defined as pathological in terms of the criteria above are denoted by an asterisk. Results are presented in detail to allow the possible application of different criteria by other researchers. The case results have been ranked and grouped according to the range of deficits the patients showed. In table 2, UA indicates that a particular subject was unable to attempt a particular test because of their level of cognitive deficit. For example, testing was abandoned for cases 19,20 , and 27 on the WCST when it became clear that they were unable to understand the nature of the test and were sorting cards at random. NC denotes a test not completed for reasons not indicative of cognitive impairment. Two cases, 26 and 9, are grouped together and were labelled "unclassifiable" because of incomplete testing. Case 26 displayed clear signs of dysmnesia but was unwilling to complete testing on the WCST. Case 9 had a history of poor social adjustment starting after the operation, but showed no evidence of intellectual or memory decline. She was, however, unable to undertake the WCST for reasons unrelated to any neuropsychological impairment.

The first six patients listed in table 2 were classified as having test results suggesting "pervasive global impairment". All six either could not undertake or performed poorly on the WCST. The first four cases showed marked deficits on the WMS, having scores well below their IQ estimates and more than one standard deviation below the mean of the WMS. The

Table 2 Individual test results for the ACoA patient group ( $n=27)$

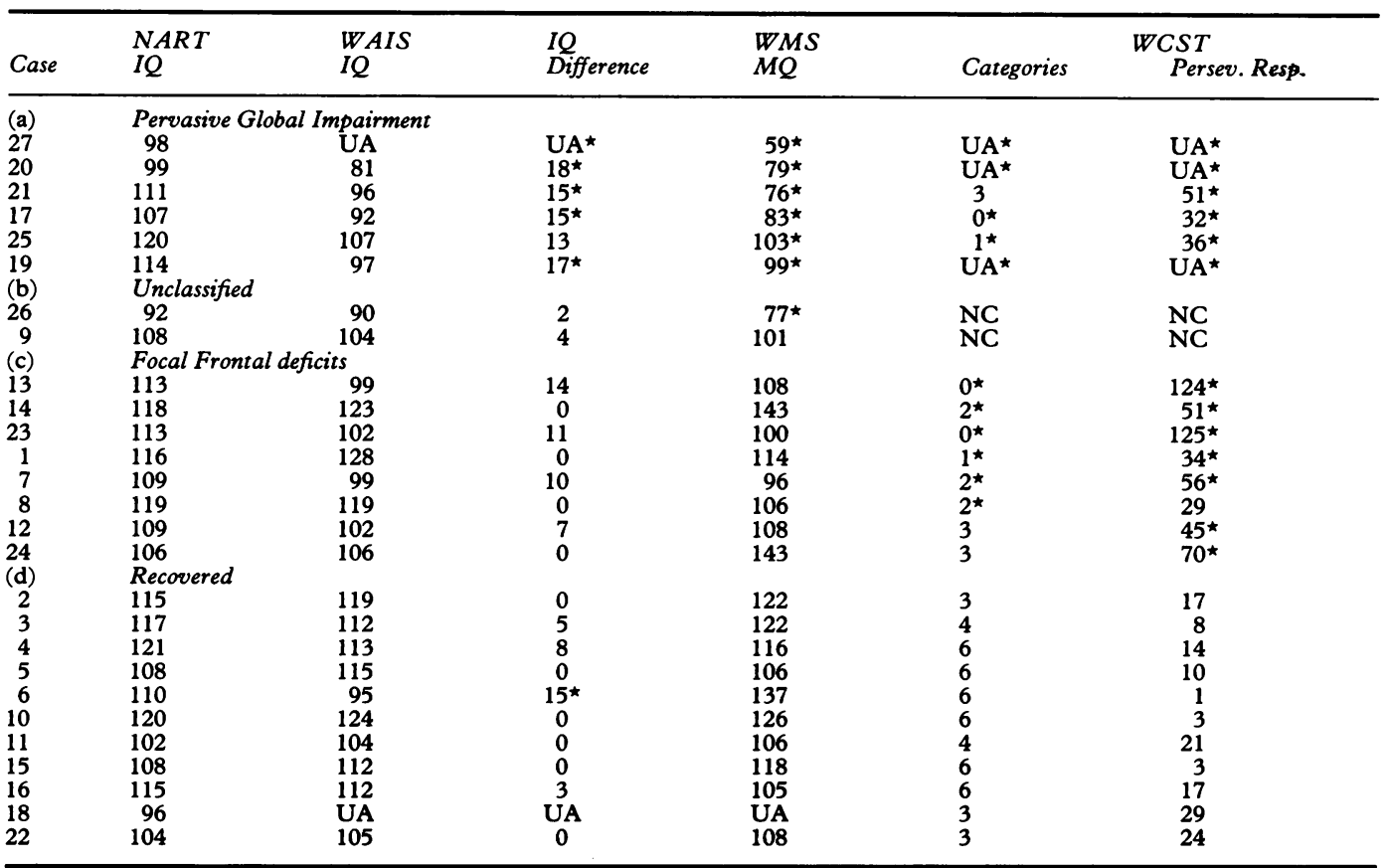


NART IQ - WAIS IQ differences were also significant in four of the cases, approached significance for case 25 , and were undetermined for the patient who presented clinically as the most severely impaired (case 27). A second group of six patients (cases 13 to 8 ) showed evidence of deficit on the WCST. There is considerable evidence available to suggest that poor performance on this test is indicative of frontal lobe damage, ${ }^{30} 31$ therefore, this group comprised subjects who might be described as: "Impaired with signs of focal frontal lobe damage". Interestingly, three of these patients reported significant memory problems during the period of their recovery. Case 7, for example, was unable to return to his job as a truck driver because of his inability to remember schedules of deliveries. Case 1 had considerable difficulty learning to find his way about in new surroundings. Two further patients (cases 12 and 24) performed poorly on the WCST, making a large number of perseverative responses but mastering at least three category changes. For both these patients, there were family or clinical reports of memory impairment during recovery, but no evidence of memory failure on the WMS. Of these eight patients, six had CT-determined evidence of infarction in the frontal lobes at the time of surgery. In contrast only one of the 11 patients designated below as recovered had similar evidence of frontal lobe infarction. Finally, there was a group of 11 patients for whom there was no evidence of impairment on formal testing or from informal clinical report; these subjects were designated as "Recovered". Unlike the preceding group of patients, only one of the unimpaired subjects had evidence of a frontal lobe infarction in the CT scan at the time of surgery.

There were no significant correlations between level of deficit and either age or length of time since surgery. The cognitive outcome data were further examined to determine the effects of other potential causes of neurological damage. Tables 3 (a), (b), and (c) report ages, preoperative clinical grades, degrees of preoperative cerebral vasospasm, and postoperative complications for 24 of the patients who completed the full test battery. Cases are arranged according to cognitive outcome as defined above: Table 3 (a) Pervasive Cognitive Impairment; Table 3 (b) Presumed Focal Frontal Lobe Impairment; and Table 3 (c) Recovered.

It is clear from column 3 of the tables that there was no relationship between clinical grade immediately before surgery and cognitive outcome in our sample. However, when operation delay due to an unstable clinical state was examined a clear relationship emerged. In five of the six patients with pervasive global impairment, surgery had been delayed until the patient's condition was stable, whereas this had occurred in only three of eight cases with conceptual learning impairment and in one of ten unimpaired cases. These proportions differed significantly, chi-square $=8.61$, $\mathrm{p}<0.025$.

Examination of column 4 of tables 3 (a), (b),

Table 3(a) Cases of pervasive global cognitive impairment

\begin{tabular}{lllll}
\hline Case & Age & $\begin{array}{l}\text { Hunt and Hess } \\
\text { grade }\end{array}$ & $\begin{array}{l}\text { Cerebral Vasospasm } \\
\text { (severity and distribution) }\end{array}$ & $\begin{array}{l}\text { Post-Operative } \\
\text { complications }\end{array}$ \\
\hline 17 & 58 & 2 & mild, focal & wound infection \\
19 & 38 & 2 & severe, diffuse & nil \\
20 & 40 & 2 & severe, diffuse & hydrocephalus \\
21 & 50 & 3 & severe, focal & nil \\
25 & 68 & 1 & nil & hydrocephalus \\
27 & 62 & 1 & mild, focal & hyd
\end{tabular}

*Vasospasm was recorded as severe only if the diameter of vessels was reduced by more than $50 \%$.

Table 3(b) Cases of cognitive impairment suggesting focal frontal lobe lesions

\begin{tabular}{rllll}
\hline Case & Age & $\begin{array}{l}\text { Hunt and Hess } \\
\text { grade }\end{array}$ & $\begin{array}{l}\text { Cerebral Vasospasm } \\
\text { (severity and distribution) }\end{array}$ & $\begin{array}{l}\text { Post-Operative } \\
\text { complications }\end{array}$ \\
\hline 1 & 47 & 2 & mild, multi-focal & extradural haematoma \\
7 & 45 & 3 & severe, diffuse & hydrocephalus \\
8 & 57 & 2 & mild, diffuse & nil \\
12 & 62 & 1 & nil & nil \\
13 & 58 & 1 & nil & nil \\
14 & 64 & 1 & nil & nubdural haematoma \\
23 & 64 & 2 & nild, focal & nil \\
24 & 70 & 2 & &
\end{tabular}

Table 3(c) Cases with no measured cognitive impairment

\begin{tabular}{lllll}
\hline Case & Age & $\begin{array}{l}\text { Hunt and Hess } \\
\text { grade }\end{array}$ & $\begin{array}{l}\text { Cerebral Vasospasm } \\
\text { (severity and distribution) }\end{array}$ & $\begin{array}{l}\text { Post-Operative } \\
\text { complications }\end{array}$ \\
\hline 2 & 67 & 3 & nil & nil \\
3 & 54 & 1 & nil & nil \\
4 & 69 & 3 & nil & nil \\
5 & 63 & 2 & nil & nil \\
6 & 51 & 2 & nil & wound infection \\
10 & 64 & 2 & nil & nil \\
11 & 45 & 2 & severe, multifocal & nil \\
15 & 33 & 1 & nil & nil \\
16 & 66 & 2 & nil & nil \\
18 & 51 & 2 & severe, focal & nil \\
22 & 41 & 2 & nil & \\
\hline
\end{tabular}


and (c) suggests a strong relationship between the presence of preoperative cerebral vasospasm, observed in angiography, and cognitive outcome. Angiographic studies were conducted on average seven days (range 2-11 days) after the haemorrhage. Those patients whose cognitive outcome was poor were more likely to have had cerebral vasospasm (five of six cases), than were those with conceptual learning impairments (four of eight cases), or those without deficits (one of ten cases). These proportionsdifferedsignificantly, chi-square = $11.31, \mathrm{p}<0.005$. The size of this sample was too small to determine the influence of severity and distribution of the vasospasm.

Postoperative complications were reported in four of the twenty five cases and hydrocephalus treated by ventriculo-peritoneal shunting developed in three patients. These complications are noted in column five of tables 3 (a), (b), and (c). The relationship between cognitive outcome and such complications is not clear in our sample. What is apparent, however, is that cerebral vasospasm, with or without other complications, is associated with cognitive impairment at long term follow up.

To illustrate the range of neurological damage and surgical complications amongst the three identifiable groups (Pervasive Cognitive Impairment, Presumed Frontal Lobe Impairment, and Recovered), three case histories are presented.

Case 20 (Pervasive cognitive impairment): This 40 year old widow sustained a coma producing subarachnoid haemorrhage. An unenhanced CT head scan performed the following day revealed a relatively thick layer of blood in all the basal cisterns. On day 6 angiography was carried out. This demonstrated a medium sized multilobular anterior communicating aneurysm projecting forwards. There was severe and diffuse spasm. By day 15, communicating hydrocephalus was revealed by the CT scan. The aneurysm was clipped without incident on day 20 post haemorrhage and external ventricular drainage was established and continued postoperatively for five days. Subsequent angiography 18 days after the operation showed satisfactory obliteration of the aneurysm and confirmed that the pre-existing vasospasm had resolved completely. A subtle decrease in her performance ability, less spontaneity, and easy distractibility just before discharge caused some concern and a predischarge CT scan showed the ventricular size to be greater than at any time previously. On day 47 a ventriculoperitoneal shunt was established. The patient recovered well from this operation and was discharged three weeks later.

Case 23 (Presumed frontal lobe impairment): This 64 year old retired man developed a coma producing subarachnoid haemorrhage. The CT scan on day 2 revealed a sausage-shaped $5 \times 2 \times 1.5 \mathrm{~cm}$ intracerebral haematoma in the infero-medial part of the right frontal lobe, surrounded by a rim of hypodensity. There was also a thick layer of subarachnoid blood. Angiography on day 9 revealed a medium sized anterior communicating aneurysm. The only significant spasm was in the right Al segment of the anterior cerebral artery. On day 10 posthaemorrhage the aneurysm was clipped without incident. The right frontal pole was excised and the haematoma evacuated. Three days later a postoperative subdural haematoma was evacuated. The patient improved over the next two weeks and was discharged five weeks later.

Case 3 (Good recovery): This 54 year old foreman was admitted to hospital after a subarachnoid haemorrhage that did not cause a coma. The CT scan on day 2 revealed a diffuse but thin layer of subarachnoid blood. Angiography on day 10 revealed an almost rounded medium-sized anterior communicating aneurysm projecting forwards and downwards. There was no spasm. The aneurysm was clipped without incident on day 14 post haemorrhage. The postoperative recovery was uneventful.

\section{Discussion}

Results from the neuropsychological assessment of the present series, confirm that longterm cognitive deficits do occur in a significant proportion of patients who have surgical repair of ACoA aneurysm rupture. In our sample of 27 patients, a total of $11(41 \%)$ showed no demonstrable evidence of impairment and could be designated as having made an excellent recovery. The patients' reports, clinical examination, and neuropsychological testing suggested that they had returned to their premorbid level of functioning, and had suffered no social, psychological, or cognitive consequences as a result of their subarachnoid haemorrhage and its treatment. Indeed, all 11 patients were able to resume their premorbid activities. These results are consistent with previous reports describing the range of outcome following ACoA aneurysmal rupture. ${ }^{10-162425}$

On average, the ACoA ruptured aneurysm patients showed a greater degree of intellectual and memory deterioration than the control group and their performance was poorer on the WCST, a measure of conceptual learning. In previous research there has been considerable focus on the presence of amnesia in cases. Three of our cases scored below 79 on the WMS, within the range usually indicative of severe memory impairment, and typical of the scores of alcoholic Korsakoff patients. ${ }^{32}{ }^{33}$ For example, the average $M Q$ score of a group of non-demented Korsakoff patients tested as part of our research programme ${ }^{32}$ was 76 . However, it is clear that specific dense amnesia, uncomplicated by other cognitive changes, is unusual in cases of ACoA aneurysm rupture. Severe amnesia more typically occurs in the presence of concurrent and often severe intellectual decline, as is apparent for the three impaired cases in our sample, and the samples of ACoA aneurysm amnesics reported by other research workers. $^{101215} \mathrm{~A}$ mild to moderate degree of memory impairment, frequently complicated by signs of frontal lobe damage (for example, 
poor WCST scores), is more typical of aneurysmal cases. This conclusion is also consistent with data reported by Mishkin ${ }^{21}$ demonstrating that monkeys with basal forebrain lesions show memory impairments, but that these are not as severe as for monkeys with specific bilateral temporal lobe or diencephalic lesions.

Analysis of the individual results revealed two putative groups of impaired subjects. The first group showed global impairment and their case notes revealed a greater incidence of vasospasm. The patients in this group did not make a good recovery and most showed the signs of poor social adjustment or cognitive decline in their everyday lives. In three patients there was moderate or severe impairment of social functioning; in four others, emotional or personality changes were reported including lability, disinhibition, and inconsistent motivation. The second group performed poorly on the WCST. This well known test is a particularly useful measure of perseveration and failure to shift mental sets, deficits found to be particularly characteristic of patients with diffuse or frontal lobe lesions. Patients in the aneurysmal sample were particularly deficient on this test. In all, 14 of the 25 surgical patients who attempted this test were impaired.

There are several potential causes of cerebral damage after ACoA aneurysm rupture and repair. These include: generalised vasospasm with ischaemia or infarction, haemorrhagic infarction of the frontal lobe following retraction during surgical intervention, and herniation of the mesial temporal lobes as a result of post operative brain swelling and hydrocephalus. ${ }^{1933}$ The most frequent mechanism of cerebral damage in our sample was ischaemia presumably resulting from vasospasm. The data reported here add to the accumulating evidence that suggests that vasospasm alone can produce long term cognitive impairment. ${ }^{1633}$ Our sample was too small to comment on the effects of the other potential mechanisms with any certainty.

Ischaemia from vasospasm does not always cause the complete destruction of cortical and subcortical matter typically observed after arteria ${ }^{*}$ occlusion, ${ }^{20}$ but will cause neuronal damage by depressing metabolism. ${ }^{34}$ Therefore, neurological damage sufficient to cause global cognitive impairment may not always be seen on CT scan. ${ }^{35}$

Our results suggest that after memory deficits have resolved, substantial impairment of function attributable to frontal lobe damage may remain. This is much more likely when there has been cerebral vasospasm in vessels supplying this part of the brain.

1 Vilkki J, Holst P, Ohman J, Servo A, Heiskanen $O$ Cognitive deficits related to computed tomographic Cognitive deficits related to computed tomographic findings after surgery for a ruptured
aneurysm. Neurosurgery 1989;25:166-72.

2 Romner B, Sonesson B, Ljunggren B, Brandt L, Saveland $\mathrm{K}$, Holtas $\mathrm{S}$. Late magnetic resonance imaging related to neurobehavioral functioning after aneurysmal subarachnoid hemorrhage. Neurosurgery 1989;25:390-7.

3 Levin HS, Goldstein FC, Ghostine SY, Weiner RL, Crof ford MJ, Eisenberg HM. Hemispheric disconnection syndrome persisting after anterior cerebral artery syndrome persisting after anterior cerebral
aneurysm rupture. Neurosurgery 1987;21:831-8.

4 Ljunggren B, Sonesson B, Saveland H, Brandt L. Cognitive impairment and adjustment in patients with neurological deficits after aneurysmal $\mathrm{SAH}$ and early operation. $J$ Neurosurg 1985;62:673-9.

5 Sonesson B, Ljunggren B, Saveland H, Brandt L. Cognition and adjustment after late and early operation for ruptured aneurysm. Neurosurgery 1987;21:279-87.

6 Saveland H, Sonesson B, Ljunggren B, Brandt L, Uski T, Zygmunt S, Hindfelt B. Outcome evaluation followin subarachnoid hemorrhage. J Neurosurg 1986;64:191-6.

7 Bornstein RA, Wer BKA, Petruk KC, Disney LB. Neuropsychological function in patients after subarachnoid hemorrhage. Neurosurgery 1987;21:651-4

8 Logue V, Durward M, Pratt RTC, Piercy M, Nixon WLB. The quantity of survival after rupture of an anterior The quantity of survival after rupture of an ant

9 Hori S, Suzuki J. Early and late results of intracranial direct surgery of anterior communicating artery aneurysms. $J$ Neurosurg 1979;50:433-40.

10 Damasio AR, Graff-Radford NR, Eslinger PJ, Damasio M Kassell N. Amnesic following basal forebrain lesions. Arch Neurol 1985;42:263-71.

11 Lindquist G, Norlen G. Korsakoff's syndrome after operation on the ruptured aneurysm of the anterior communicating artery. Acta Psychiat Scand 1966;42:234.

12 Alexander MP, Freedman M. Amnesia after anterior communicating artery aneurysm rupture. Neurology 1984 34:752-7.

13 Gade A. Amnesia after operations on aneurysms of the anterior communicating artery. Surg Neurology 1982; 18:46-9.

14 Parkin AJ, Leng NR, Stanhope N, Smith AP. Memory impairment following ruptured aneurysm of the anterior impairment following ruptured aneurysm of the ant

15 Richardson JTE. Performance in free recall following rupture and repair of intracranial aneurysm. Brain and $C o$ 1989;9:210-26.

16 Vilkki J. Amnesic syndromes after surgery of anterior communicating artery aneurysms. Cortex 1985;21:431-44.

7 Perlmutter D, Rhoton AL, Microsurgical anatomy of the anterior cerebral-anterior communicating recurrent artery complex. $J$ Neurosurg $1976 ; 45: 259-72$.

18 Crowell RM, Morawetz RB. The anterior communicating artery has significant branches. Stroke 1977;8:272-3.

19 Yasargil MG, Smith RD. Management of aneurysms of anterior circulation by intracranial procedures. In: Youmans JR, ed. Neurological neurosurgery, vol 3. Philadelphia: WB Saunders, 1979.

20 Bouillin DJ, ed. Cerebral vasospasm. Chichester: Wiley, 1980.

21 Mishkin M, Malamut B, Bechevalier J. Memories and habits. Two neural systems. In: Lynch G, McGaugh JL, memory. New York. Guildford Press, 1984:65-77.

22 Markowitsch HJ, Pritzel M. The neuropathology of amnesia. Progress in Neurobiology 1985;25:189-287.

23 Corkin S, Cohen NJ, Sullivan EV, Clegg RA, Rosen TJ Ackerman RH. Analyses of global memory impairment of different etiologies. Annals of the NY Acad of Sciences 1985;444:10-40

24 Sengupta RP, Chiu JSP, Brierley H. Quality of surviva following direct surgery for anterior communicatin artery aneurysms. $J$ Neurosurg 1975;43:58-64.

25 du Cros JT, Lhermitte F. Neuropsychological analysis of rupture saccular aneurysms of the anterior communicating artery after radical therapy ( 32 cases). Surg Neurol ing artery after

26 Hunt WE, Hess RM. Surgical risk as related to the time of intervention in the repair of intracranial aneurysms. Neurosurg 1968;28:14-20.

27 Nelson H. Manual for the National Adult Reading Test. Windsor, UK: NFER-Nelson, 1982.

28 Wechsler D. Manual for the Wechsler Adult Intelligence Scale. NY: The Psychological Corporation, 1955

29 Wechsler D. A standardized memory scale for clinical use. $J$ Psychol 1945;19:87-95.

30 Heaton RK. A Manual for the Wisconsin ${ }^{\circ}$ Card Sorting Test. Odessa, FL: Psychological Assessment Resources, 1981.

31 Lezak M. Neuropsychological assessment, 2nd ed. NY Oxford University Press, 1983

32 Longmore BE, Knight RG. The effect of intellectua deterioration on retention deficits in amnesic alcoholics. Abnorm Psychol 1988;97:448-54.

33 Squire LR. Comparisons between forms of amnesia: Some deficits are unique to Korsakoff's syndrome. JExp Psychol: Learning, Memory and Cognition 1982;8:560-71.

34 Grubb RL, Raichle ME, Eichliy JO, Gado MH. Effects of subarachnoid haemorrhage on cerebral blood volume,
blood flow, and oxygen utilization in humans. J Neurosurg blood flow, and

35 Volpe BT, Hirst W. Amnesia following the rupture and repair of an anterior communicating artery aneurysm. $J$ repair of an anterior communicating artery
Neurol Neurosurg Psychiatry 1983;46:704-9. 